\title{
Design and Implementation of Emotional Virtual Experiment User Model Based on VRP
}

\author{
Linlin GAO \\ Department of Quality Control Office, Yunnan Open University, Kunming Yunnan, China 650000 \\ Email: 382082609@qq.com
}

Keywords: Virtual Experiment, Emotional Design, VRP

\begin{abstract}
With the development of science and technology as well as educational technology, people are paying more and more attention to the positive influence of emotional, humanistic, aesthetic and other elements of virtual experiment on the cognitive psychology of learners. The emotional design of virtual experiment system changes the emotional demand of learners to design element of virtual experiment system, and then brings about physiological and psychological satisfaction and pleasure to learners. This paper has proposed the program of emotional virtual experiment user model designed and developed by 3Ds Max and VRP by taking the emotional design of Norman as theoretical basis and discussed the feasibility of program by taking experiment as example.
\end{abstract}

\section{Introduction}

It is well known that as a new experimental teaching method, virtual experiment is playing a more and more important role in practice teaching. But many virtual experiment systems at present have problems of too stiff, not vivid and can't arouse the interest or enthusiasm of learners etc, therefore, the writer has introduced the emotional design theory of Norman into virtual experiment, established brand new emotional virtual experiment user model and discussed its feasibility by taking the virtual experiment made by writer as example.

\section{The Emotional Design Theory of Norman}

Cognitive psychologist Donald A A Norman has put forward three design levels of instinct, behavior and reflection of design in his "Design Psychology 3: Emotional Design".

The instinct level design is related to the first reaction of people and feeling, sound and other physiological characteristics play a leading role; the best situation that instinct layer design needs to achieve is that when people see the design at first glance, they can't help to produce strong desire of attaining and gaining. The design of this layer pays great attention to the product appearance.

The behavior level design is related to use and the most important is the realization of function. The first step of good behavior level design is to understand how does the customer use product, so studying the using habit and behavior model of users for products is very important and usability also becomes the key test factor of products.

The reflection level design pays attention to the overall impression of products and focuses on the good memory brought about by design as well as the self-realization of demand level attained from design.

\section{Emotional Virtual Experiment User Model}

Based on the emotional design concept of Norman, the writer has combined emotional design and virtual reality technology together and constructed an emotional virtual experiment user model which is application-oriented and takes the emotional cognition of users as the center by starting from the research on emotional elements of three design level and fully considering about the characteristics of virtual experiment environment. 
This model has transferred the three levels of emotional design objectives of Norman to details in design, which has put emphasis on instinctive reaction and perception of users, using habit of learners, functional realization of virtual experiment, the overall image and self-realization used by learners. The model is as shown in following picture 1 :

\begin{tabular}{|c|c|c|c|c|c|c|c|c|c|c|c|c|c|c|c|c|c|c|}
\hline 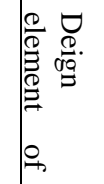 & $\stackrel{\curvearrowright}{\varrho}$ & & $\begin{array}{l}3 \\
0 \\
0 \\
\frac{1}{0} \\
\frac{1}{10}\end{array}$ & 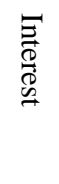 & & 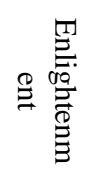 & 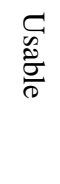 & & 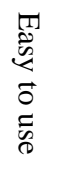 & 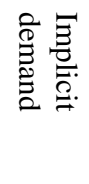 & & 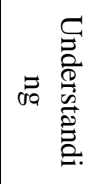 & 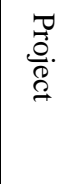 & & ఖ్రి & 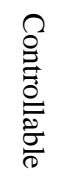 & & 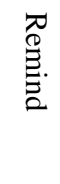 \\
\hline & 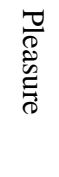 & 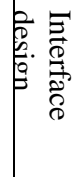 & & & 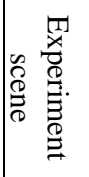 & & & 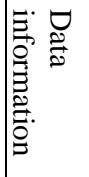 & & & 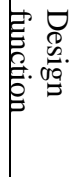 & & & 管 & & & 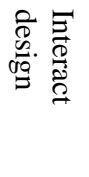 & \\
\hline 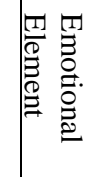 & 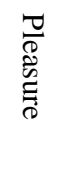 & & 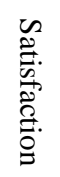 & 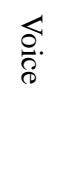 & & 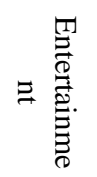 & 焉 & & $\begin{array}{l}5 \\
0 \\
0 \\
0 \\
0 \\
0 \\
0\end{array}$ & 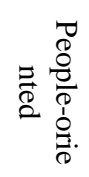 & & 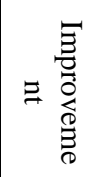 & 岂 & & 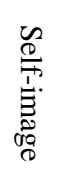 & 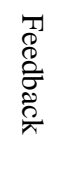 & & 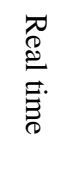 \\
\hline & & 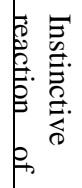 & & & 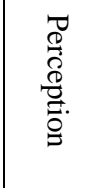 & & & 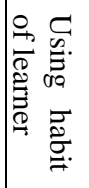 & & & 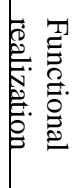 & & & 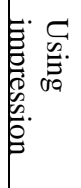 & & & 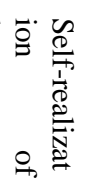 & \\
\hline 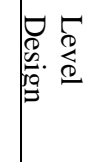 & 莺 & & & & & & 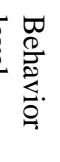 & & & & & & 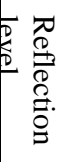 & & & & & \\
\hline
\end{tabular}

fig 1 Emotional Virtual Experiment User Model

\section{Design and Application of Emotional Virtual Experiment User Model}

As shown in picture 1, this user model has transferred the emotional element of instinct level, behavior level and reflection level into the design elements of virtual experiment and paid attention to the design details of six aspects: interface design, experiment scene design, data information design, design function, virtual situation design and fault tolerant interaction design.

Interface Design

Interface design mainly takes realizing the emotional objective of instinct level for user as basis, pays attention to the instinctive reaction and perception of leaner; color, modeling, pleasure, satisfaction and other elements become the keys of interface design.

Beautiful composition and color matching become important elements here. In virtual experiment, it needs to pay attention to the consistency of color and learning content, so the experiment can not only transfer information in a scientific way, but also produce strong visual attack, such as adopting dark blue, blue and other cold colors for education and science and technology subjects to present the quiet, profound and other perceptions. The layout is better to be brief and there should not be too much objects in the same picture, which can highlight the focus and attract the learning attention of learners. For example, it adopts left and right frame type in this experiment, the right is the reminding buttons for experiment operation and the right presents experiment content, displaying clear, standardized and organized structure. 

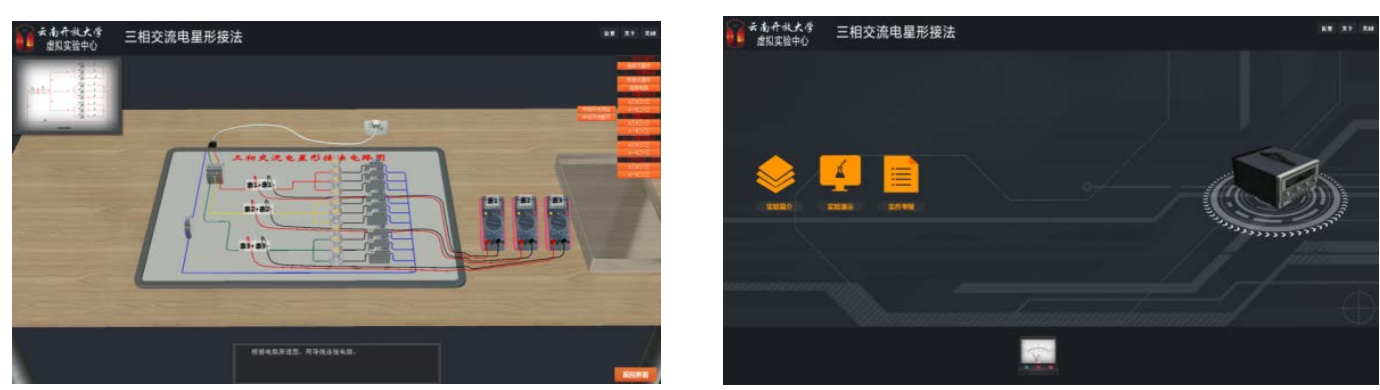

Experiment Scene Design

fig 2 Interface Design of Virtual Experiment

Experiment scene design mainly takes realizing the emotional objective of instinct level for user as basis; interest, enlightenment, sound, entertainment and other elements become the keys of experiment scene design.

As shown in picture 3, this virtual experiment takes real laboratory as experiment scene, changes the boring buttons into interesting and cartoon icons, which can stimulate the desire of users to explore and motivate them to explore. The implementation of order for each step is with voice commentary.

Data Information Design

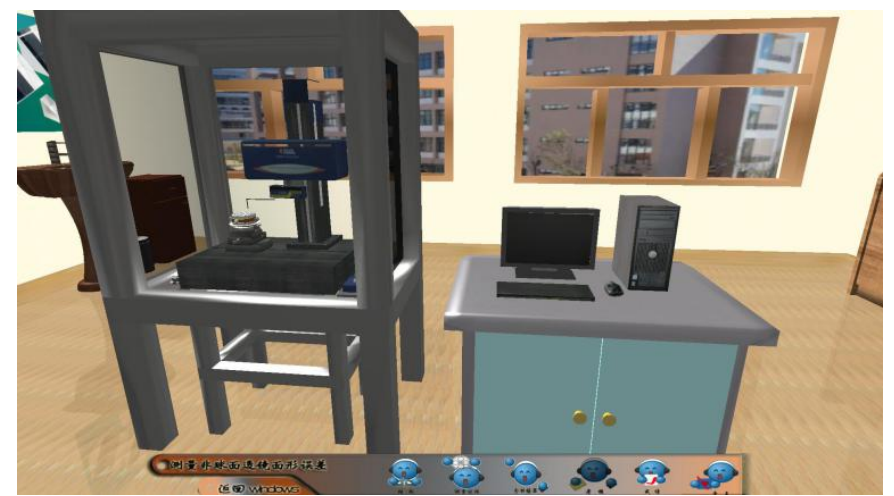

fig 3 Experiment Scene Design of Virtual Experiment

Data information design mainly takes realizing the emotional objective of behavior level for users as basis, puts emphasis on functional realization of using habit and virtual experiment of learners; usable, easy to use, high efficiency and less error are key factors of data information design.

As shown in picture 4, the first level interface of this experiment adopts very simple thought, second level button and third level button is put on the upper right part in a simple and clear way, achieving usable, easy to use, high efficiency and less error effect, put emphasis on the using habit of learners.

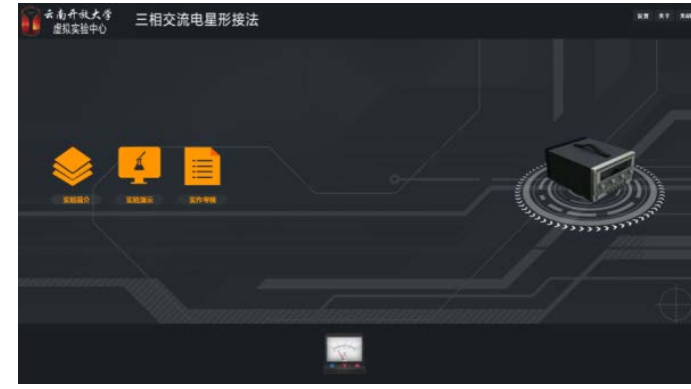

fig 4 Data Information Design of Virtual

Design Function

Design function mainly takes realizing the emotional objective of behavior level for users as basis, pays attention to the using habit of learners and functional realization of virtual experiment; implicit demand, understanding, people-oriented and improvement are key factors of design function.

Good behavior level design should be people-oriented, focusing on understanding and satisfying 
people who use the product in a genuine way. The design team of virtual experiment needs to produce the product prototype quickly for potential users to try out. With the continuation of design process, the attained information in the test will be integrated. When the product is accomplished, it already passed complete using inspection and this repeated design process is effective and takes user as the core of the design.

Virtual Scene Design

Virtual scene design mainly takes realizing the emotional objective of reflection level for users as basis, puts emphasis on overall impression and self-realization of using for learners; project, case, task and self-image are key factors of virtual scene design.

As shown in picture 5, "virtual experiment of milling technology" belongs to on of the projects of "Optical Parts Processing" course. Virtual experiment of milling technology adopts task completion and divides the main menu into five parts: "see what it is", "guess why", "try how to do", "have you got it" and "let's interact with each other". Learners participate as hosts-explore, accomplish task and shape own image.

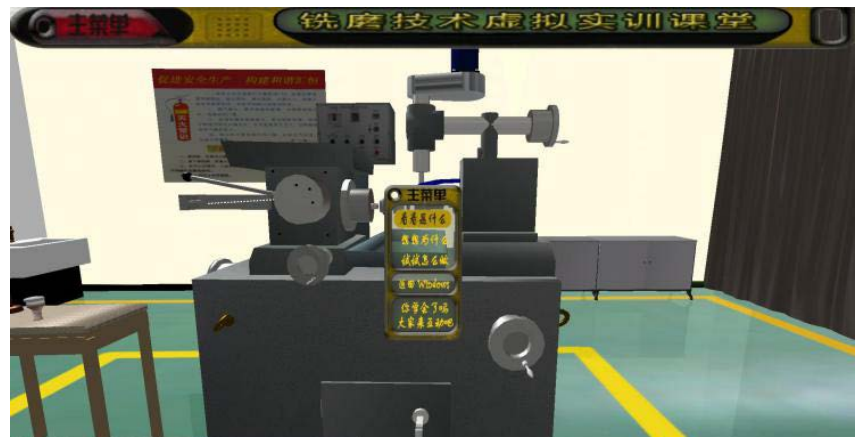

fig 5 Virtual Scene Design of Virtual Experiment

Fault Tolerant Interaction Design

Fault tolerant interaction design mainly takes realizing emotional objective of reflection level for users as basis, puts emphasis on overall impression and self-realization of using for learners; controllable, reminding, feedback and real time are key factors of interaction design. The focus of fault tolerant interaction design is respecting users and their objectives and exploring the dialogue between virtual experiment products and users. Virtual experiment is requested to provide real time reminding, real time error reminding and real time feedback to learners and the learners can select study content, control experiment process and test independently in the using process.

\section{Conclusion}

In the era of network technology, virtual experiment makes new attempt for experiment teaching method. In the people-oriented perception economic era, the humanized and emotional design injects new design concept to virtual experiment and provides new ideas to users in improving their self-learning ability at the same time. Emotional virtual experiment respects users and their objectives, explores the dialogue between virtual experiment products and users and draws the distance between users and virtual experiment products closer.

\section{Acknowledgement}

Yunnan Provincial Department of Education Science Research Fund general project(2014Y366). "Based on the VRP 3D virtual laboratory construction".

\section{Reference}

[1] Jinyu Hu and Zhiwei Gao. Distinction immune genes of hepatitis-induced heptatocellular carcinoma[J]. Bioinformatics, 2012, 28(24): 3191-3194.

[2] Ying Liang, Peiyi Lu.(2014). Medical insurance policy organized by Chinese government and 
the health inequity of the elderly: longitudinal comparison based on effect of New Cooperative Medical Scheme on health of rural elderly in 22 provinces and cities, International Journal for Equity in Health, 13:37, 1-15.

[3] Jinyu Hu, Zhiwei Gao and Weisen Pan. Multiangle Social Network Recommendation Algorithms and Similarity Network Evaluation[J]. Journal of Applied Mathematics, 2013 (2013).

[4] Ying Liang, Peigang Wang. (2014). Influence of Prudential Value on the Subjective Well-Being of Chinese Urban-Rural Residents, Social Indicators Research, 118(3): 1249-1267. 